University of Nebraska - Lincoln

DigitalCommons@University of Nebraska - Lincoln

Faculty Publications from the Department of

Electrical and Computer Engineering

Electrical \& Computer Engineering, Department

8-14-2018

\title{
Development of boron calibration via hybrid comparator method in prompt gamma activation analysis
}

\author{
E. J. Artnak
}

S. R. Biegalski

S. Landsberger

Natale lanno

Dennis Alexander

See next page for additional authors

Follow this and additional works at: https://digitalcommons.unl.edu/electricalengineeringfacpub

Part of the Computer Engineering Commons, and the Electrical and Computer Engineering Commons

This Article is brought to you for free and open access by the Electrical \& Computer Engineering, Department of at DigitalCommons@University of Nebraska - Lincoln. It has been accepted for inclusion in Faculty Publications from the Department of Electrical and Computer Engineering by an authorized administrator of DigitalCommons@University of Nebraska - Lincoln. 


\section{Authors}

E. J. Artnak, S. R. Biegalski, S. Landsberger, Natale lanno, Dennis Alexander, and M. F. Byers 


\title{
Development of boron calibration via hybrid comparator method in prompt gamma activation analysis
}

\author{
E. J. Artnak, ${ }^{1}$ S. R. Biegalski, ${ }^{1,2}$ S. Landsberger, ${ }^{1}$ \\ N. J. Ianno, ${ }^{3}$ D. Alexander, ${ }^{3} \&$ M. F. Byers ${ }^{1}$ \\ 1 Nuclear Engineering Teaching Laboratory, The University of Texas at Austin, \\ 10100 Burnet Road, Building 159, Austin, TX 78758, USA \\ 2 Nuclear and Radiological Engineering and Medical Physics Programs, Georgia \\ Institute of Technology, 770 State Street, Atlanta, GA 30332, USA \\ 3 Department of Electrical and Computer Engineering, University of Nebraska-Lincoln, \\ 844 N 16th Street, Lincoln, NE 68588, USA \\ Corresponding author — E. J. Artnak, UT@att.net
}

\begin{abstract}
The prompt gamma activation analysis (PGAA) facility at the Nuclear Engineering Teaching Laboratory at The University of Texas at Austin was utilized to quantify boron concentrations in boron carbide semiconductor films deposited on silicon substrates. Calibration was complicated by the unique and varying sample geometries analyzed. In addition, there was a dearth of solid materials available with quantified boron concentrations having comparable or readily modifiable dimensions to exploit for calibration purposes. Therefore, a novel hybrid comparator method was developed for the quantification of boron utilizing aluminum as an inexpensive and easily machinable reference material. Aluminum samples were manufactured with high tolerances to match the geometry of each sample of interest. Each boron carbide film sample and its congruent aluminum sample were measured in the PGAA system. The measured aluminum responses and relevant nuclear parameters were used to standardize the
\end{abstract}

Published in Journal of Radioanalytical and Nuclear Chemistry 318 (2018), pp 271-277. doi 0.1007/s10967-018-6062-3

Copyright (C) 2018 Akadémiai Kiadó, Budapest, Hungary; published by Springer. Used by permission.

Submitted 12 April 2018; published 14 August 2018. 
measurements. A boron standard was created using a procedure derived from a similar approach used by the National Institute of Standards and Technology. Quality control measurements using this standard show that the method provided accuracy to within $5 \%$ for boron quantification.

Keywords: Prompt gamma activation analysis (PGAA), Boron, Calibration

\section{Introduction}

Prompt gamma activation analysis (PGAA) has become a powerful nuclear analytical technique that continues to demonstrate value in an increasing number of applications [1]. The fundamentals, practice, and key advancements in applied methods of PGAA are well documented in the literature $[2,3]$. With reported detection limits of less than $10 \mathrm{ng} \mathrm{g}^{-1}$ in some materials [4], PGAA is one of the most sensitive, non-destructive analytical techniques available for low-level boron quantification. This makes PGAA a very attractive option for quantitative analysis of boron in thin-film semiconductors, where deposition characteristics and boron concentration of thin-film growth are crucial to device function.

A big challenge is obtaining an accurate characterization and efficiency calibration of the system. Absolute (parametric) and relative calibration (or standardization) methods are largely considered inaccurate or impractical in most analyses, although strict parameterization approaches have been pursued [5]. The accuracy and applicability of more appealing comparator-type and $k_{0}$-type standardization methods originating in neutron activation analysis (NAA) are well established [6-8] and consequent efforts leading to successful implementation of these calibration techniques in standard PGAA practice have been described $[9,10]$.

In a recent study, boron carbide thin film samples were analyzed for absolute boron concentrations using the PGAA facility at the Nuclear Engineering Teaching Laboratory (NETL) at The University of Texas at Austin. Calibration of the system was complex due to a number of factors, which include the following: large number of samples with different shapes and sizes, spatially nonuniform neutron flux profile at sample location [5, 11], absence of boron solid material standards with comparable geometries and properties (absorption and scattering), and a required quick turnaround with limited expense. Furthermore, at the onset of analysis, characterization of the silicon substrates on which the 
boron carbide films were deposited was limited. It was known only that boron carbide film deposition was uniform and boron concentration per unit area of film was $\leq 250 \mu \mathrm{g} \mathrm{cm}^{-2}$ for each sample. In a strict sense of their definition, neither the comparator nor the $k_{0}$ calibration method was optimal in itself for these experimental constraints.

In this paper, the boron calibration method developed and utilized in this study of boron carbide films using PGAA is presented. The validity of the results using this approach are examined through an internal comparison of known silicon content in the substrates, which was acquired only after completion of the original analysis, as well as in comparison with a carefully constructed in-house boron standard. The results confirm that this method is capable of achieving accurate measurements of boron in thin-film semiconductors at minimal material expense with promising application in other elemental analyses.

\section{Theory}

In PGAA measurements, count rates from experimentally detected gamma rays of a characteristic energy are used to determine elemental concentrations of the materials analyzed. For most PGAA facilities and practical applications, the conditions in which these measurements are made permit a number of simplifications to be made in the analysis. In this study, the boron carbide thin film and silicon substrate is treated as a homogenous volume (to be later confirmed) over the small sample thickness. Neutron self-shielding in the sample due to boron is deemed negligible as less than $1 \%$ of the neutron flux is attenuated through the sample given the maximum film thickness, maximum possible boron concentration stated, and the boron total interaction cross-section. The PGAA facility neutron flux is sub-thermal with the faster neutron component removed due to the $6 \mathrm{~m}$ long, curved neutron guide [12]. For the light elements composing each sample matrix, gamma-ray self-absorption is negligible, especially as the samples are positioned at $45^{\circ}$ with respect to the incident neutron beam and high-purity germanium (HPGe) detector face. Based on these assertions, for a given set of experimental conditions, the net peak area $A_{x}$ from a neutron capture gamma ray of energy $E_{\mathrm{c}}$ from element $x$, can be expressed as a product of constant terms $[3,9]$ : 


$$
A_{x, E_{\gamma}}=\left[\frac{N_{A} m_{x}}{M_{x}}\right] \varepsilon_{E_{\gamma} V} \theta_{x} \Gamma_{x, E_{Y}} t_{l} \Phi_{t h} g \sigma_{o, x}
$$

where $N_{A}$ is Avogadro's number, $M_{x}$ is the atomic weight, $m_{x}$ is mass of element $x, \varepsilon_{E_{Y} V}$ is the detector's efficiency within volume $V$ of sample, $\theta_{x}$ is the isotopic abundance, $\Gamma_{x,}$ is the gamma-ray yield in photons per capture, $t_{l}$ is the detector live time during irradiation, $\Phi_{t h}$ is the thermal flux, $g$ is the Westcott factor, and $\sigma_{o, x}$ is the thermal neutron capture cross-section.

In addition, the Westcott factor $g \approx 1$ for all isotopes of interest in this study and so behave as good $1 / v$ absorbers, which removes the dependence in Eq. (1). Using the definition of the partial elemental capture cross-section $\sigma_{\gamma, E_{\gamma}}^{x}$ [3], and rewriting the bracketed terms as the number density $N_{x}$ of element $x$ since the mass density $\rho_{x}$ of any element $x$ is known, Eq. (1) can be written as:

$$
A_{x, E_{\gamma}}=N_{x} \varepsilon_{E_{\gamma} V} \Phi_{t h} \sigma_{\gamma, E_{\gamma}}^{x}
$$

Normalizing the net peak area of element $x$ to a comparator element $c$, the following is obtained:

$$
\frac{\left[A_{x, E_{\gamma}} / t_{l}\right]_{x}}{\left[A_{c, E_{Y}} / t_{l}\right]_{c}}=\frac{\left[N_{x} \varepsilon_{E_{\gamma} V} \Phi_{t h} \sigma^{x}{ }_{\gamma, E_{\gamma}}\right]_{x}}{\left[N_{c} \varepsilon_{E_{\gamma} V} \Phi_{t h} \sigma_{\gamma, E_{\gamma}}^{c}\right]_{c}}
$$

As noted, the neutron flux at the sample location is spatially non-uniform, however, as long as element $x$ and comparator element $c$ are homogenously mixed in sample, then the flux cancels out of Eq. (3). This is also true if element $x$ and $c$ are in separate samples placed in the exact same location in front of the neutron beam and have similar neutron and gamma-ray self-shielding properties (each sample matrix has similar nuclear interaction compositions) and all of the above assumptions are accurate. This also removes the $V$ dependence in the efficiency term $\varepsilon$ for both elements. On a final note, the variation in flux intensity over time due to changes in the reactor operating conditions must be accounted for in every PGAA measurement as this can occur over the course of a long count and especially between day-to-day irradiations. A chief contributor to this variation is thought to be ${ }^{135} \mathrm{Xe}$ buildup in the reactor core [13]. These variations are accounted for by normalizing the 
counts in each PGAA measurement to the neutron counts acquired by a proportional ${ }^{3} \mathrm{He}$ detector located between the curved neutron guide and focusing element $[11,13]$. The neutron counts from the ${ }^{3} \mathrm{He}$ detector must also be normalized in each measurement to the HPGe detector real time during the irradiation before being directly compared to background or other comparator element measurements. As such, Eq. (3) can be rewritten and solved for the unknown atom density $N_{x}$ of element $x$ by comparison with an internal (or separate external sample of) comparator element $c$ as follows:

$$
N_{x}=N_{c} \frac{\left[\left(A_{x, E_{\gamma}} t_{r}\right) /\left(t_{l} n_{x}\right)\right]_{x}}{\left[\left(A_{c, E_{\gamma}} t_{r}\right) /\left(t_{l} n_{c}\right)\right]_{c}} \frac{\left[\varepsilon_{E_{\gamma}} \sigma_{\gamma^{\prime} E_{\gamma}}\right]_{c}}{\left[\varepsilon_{E_{\gamma}} \sigma^{x}{ }_{\gamma, E_{\gamma}}\right]_{x}}
$$

where $t_{r}$ is the HPGE detector real time during the irradiation and $n_{x}$ is the number of neutrons detected by the ${ }^{3} \mathrm{He}$ detector. Note this assumes that background contributions have already been subtracted out from the quantity represented by the terms in brackets. Equation (4) is then used to determine the unknown mass of element $x$ in all PGAA measurements, whether the comparator element is internal to the sample containing element $x$ or external in another sample of identical geometry.

In this study, both ${ }^{27} \mathrm{Al}$ and ${ }^{28} \mathrm{Si}$ are used as comparator elements to determine natural boron elemental concentrations in the boron carbide thin films and the results are compared. The ${ }^{27} \mathrm{Al}$ is present in known quantities as external comparator samples composed of 6061-T6 aluminum alloy, which are easily fabricated with identical geometry to each boron carbide sample. The ${ }^{28} \mathrm{Si}$ is present in the silicon substrates on which the boron carbide films are deposited and are treated as an internal comparator that is homogenously mixed in each sample.

The boron gamma-ray peak of interest is the $477 \mathrm{keV}$ photopeak emanated from the ${ }^{10} \mathrm{~B}(n, \alpha){ }^{7} \mathrm{Li}$ reaction, which is Doppler-broadened (460$490 \mathrm{keV}$ ) due to the recoiling ${ }^{7} \mathrm{Li}$ nucleus after alpha particle emission. This peak cannot be fit using conventional Gaussian peak-fitting software and detailed deconvolution and treatments of peak-fitting routines for the Doppler-broadened boron peak shape have been discussed in the literature $[14,15]$. However, as in the study by Harrison and Landsberger in 2009 [16] involving the determination of boron over a large dynamic range using the NETL PGAA facility, the use of a simple peak-summing technique to measure boron peak area in the current study was found to introduce less than $1 \%$ error. The major interference contributors to 
the $477 \mathrm{keV}$ boron peak are expected to be germanium $(467.6,468.1$, $468.8,470.0,472.1,475.4,476.8,479.8,481.7,484.6,488.3 \mathrm{keV}$ ) due to scattering of neutrons off the sample into the HPGe detector crystal, sodium (472.2 keV) from human contamination, and silicon (477.1 keV) from the substrate upon which the boron films are deposited. Using the cross-section ratio and net peak area at a separate, interference-free peak (different energy gamma ray) of the same element, the contribution of that element as an interference to the Doppler-broadened boron peak can easily be determined. Again, interferences were determined to be insignificant for all isotopes of interest and the use of a peak-summing technique for boron was found to be accurate in this study.

\section{Experimental}

All experiments were conducted using the NETL PGAA facility at The University of Texas at Austin. The PGAA facility is equipped with the Texas Cold Neutron Source (TCNS), which has the ability to pass neutrons streaming out of beam port \#3 on the TRIGA Mark II research reactor through a moderating chamber, which is cooled to $27 \mathrm{~K}$. However, these experiments did not require an increased sub-thermal neutron flux fraction, so the TCNS was not in operation during these experimental measurements and the moderating chamber was purged with helium instead. A $6 \mathrm{~m}$ long neutron guide with a $300 \mathrm{~m}$ radius of curvature preferentially directs low velocity neutrons toward the sample through biological shielding at a wavelength of $4.35 \AA$ and effective temperature of $39 \mathrm{~K}$ [11]. The collimated thermal-equivalent neutron flux reaching the sample position has been measured previously at $5.32 \times 10^{6} \mathrm{~cm}^{-2}$ $\mathrm{s}^{-1}$ when the reactor operates at a power of $950 \mathrm{~kW}$ [11]. The resulting gammas from the irradiated sample are measured by a horizontal, $\mathrm{p}$ type, 65\% efficient ORTEC GMX Series High Purity Germanium (HPGe) detector. Detailed Schematics and characterization of the facility including background contributions affecting detection limits are published elsewhere $[11,13]$.

While the facility is equipped with a number of different sample holding devices [17] the wide variation in size and geometry of samples required for the boron carbide semiconductor studies rendered precise and reproducible placement in the spatially non-uniform beam 
impossible. Therefore, custom sample holders were created to allow samples of varying dimensions and their geometrically analogous comparator to experience an identical incident neutron flux. The holders were fabricated out of 6061-T6 aluminum alloy with a threaded rod with screw and nut assembly body allowing sample height and angle to be adjusted as well as locked into place. Four locating pins on the base further stabilize the sample and ensure consistent placement into a fixed baseplate. Interchangeable head plates were fabricated in order to accommodate both rectangular and circular boron carbide semiconductor geometries. The head plate machined for circular geometries was designed with a concave internal surface to match the radius of curvature of circular disk samples, while a locating pin was utilized in the head plate for rectangular samples.

Comparator samples for each unique geometry were constructed out of easily machinable aluminum alloy, which is also a more-realistic and currently studied substrate material for possible large-scale production of ${ }^{10} \mathrm{~B}_{4} \mathrm{C}$ thin films used in gaseous neutron detectors [18]. The cost and material properties of aluminum allowed for inexpensive, timely construction of a large number of comparators using standard machine shop tools.

In the case of the in-house standard, a known mass of certified aqueous boron standard material (Inorganic Ventures, MSB-100PPM) was deposited onto filter paper affixed to an aluminum substrate sample, which ensured that all liquid remained on the surface and that the boron was uniformly deposited across the surface area of the sample. Excess liquid solvent was then evaporated in a vacuum oven resulting in a thin, uniformly distributed boron layer atop the aluminum substrate. This process of deposition and solvent evaporation on an absorbing material substrate is derived in part from steps used by the National Institute of Standards and Technology (NIST) to create solid boron standards in pellet form [4].

The characteristic gamma ray of interest in the PGAA measurements was ${ }^{27} \mathrm{Al}(1779 \mathrm{keV})$ for the 6061-T6 aluminum alloy comparators and ${ }^{28} \mathrm{Si}$ (3539 keV) for the silicon substrate internal comparators. It is important to note that in this study the ${ }^{27} \mathrm{Al}(1779 \mathrm{keV})$ gamma ray is not prompt; it has a 2.24 min decay. In order to account for this non-prompt gamma-ray emission, counting is delayed after opening the neutron beam shutter by a minimum of $30 \mathrm{~min}$. 


\section{Procedure}

Prior to the acquisition of PGAA sample spectra, the detector efficiency was calculated using a similar method as described in Biegalski et al. [5]. A NIST traceable ${ }^{152} \mathrm{Eu}$ point source was utilized to calculate a base efficiency curve for the sample. An iron foil was then measured in the PGAA facility and a relative efficiency curve was calculated out to $7646 \mathrm{keV}$. The ${ }^{152} \mathrm{Eu}$ and the Fe foil based efficiency curves were normalized and merged. The final efficiency fit was calculated utilizing a least-squares fitting algorithm to create a fourth-order polynomial in lognormal energy and count space. The relative uncertainty in the efficiency curve is estimated to be $\pm 1.2 \%$ based on propagation of uncertainty from the peak counting statistics, source activity uncertainty, gamma-ray yield uncertainty, and the $\chi^{2}$ value for the fit.

Depending upon the general shape of the sample, either the disk or square head plate was attached to the sample holder base plate and a background spectrum was acquired. In order to align the sample within the incident neutron beam, a mock-up replica of the general sample geometry was created using a Formlabs stereolithography (SLA) 3D printer, which features either a peripheral slot around the sample perimeter for inserting a cadmium wire or a circular inset for attaching a cadmium disk on the sample surface. Using this replicate with cadmium insert, images were captured by a neutron radiography camera placed directly behind the sample holder with the beam port shutter open as shown in Fig. 1. Adjustments were made to the sample holder until the sample was correctly aligned.

After aligning the neutron beam with a particular sample geometry, the actual boron carbide sample was placed in the sample holder and a spectrum was acquired, immediately followed by the irradiation of its corresponding aluminum comparator.

\section{Results and discussion}

A total of ten boron carbide thin-film samples, which had been deposited on silicon substrate backings, were analyzed. Another ten comparator samples composed of 6061-T6 aluminum alloy, each having identical dimensions to a boron carbide sample, were also analyzed in order to standardize the PGAA measurements for boron content. The surface 

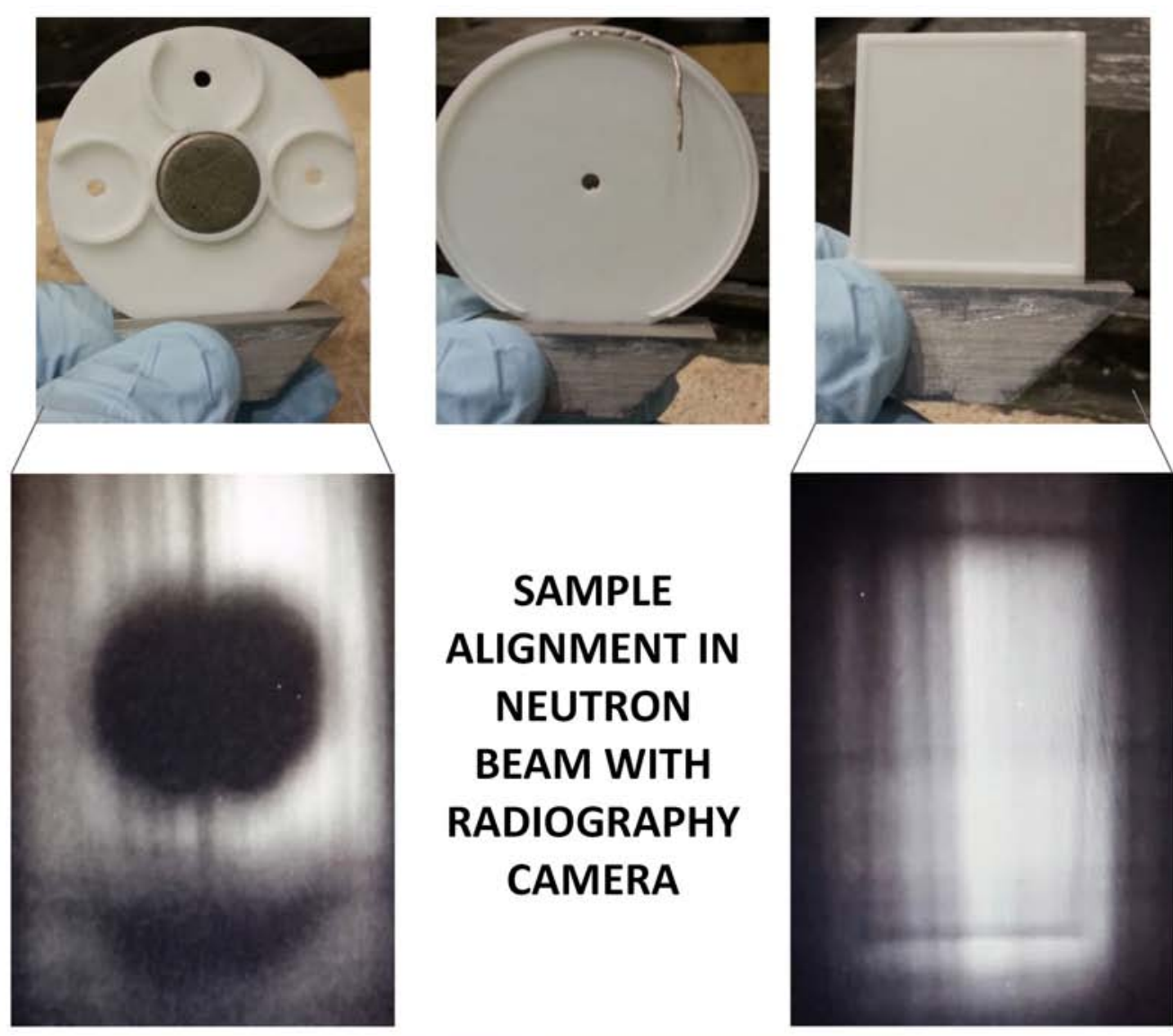

SAMPLE ALIGNMENT IN NEUTRON BEAM WITH RADIOGRAPHY CAMERA

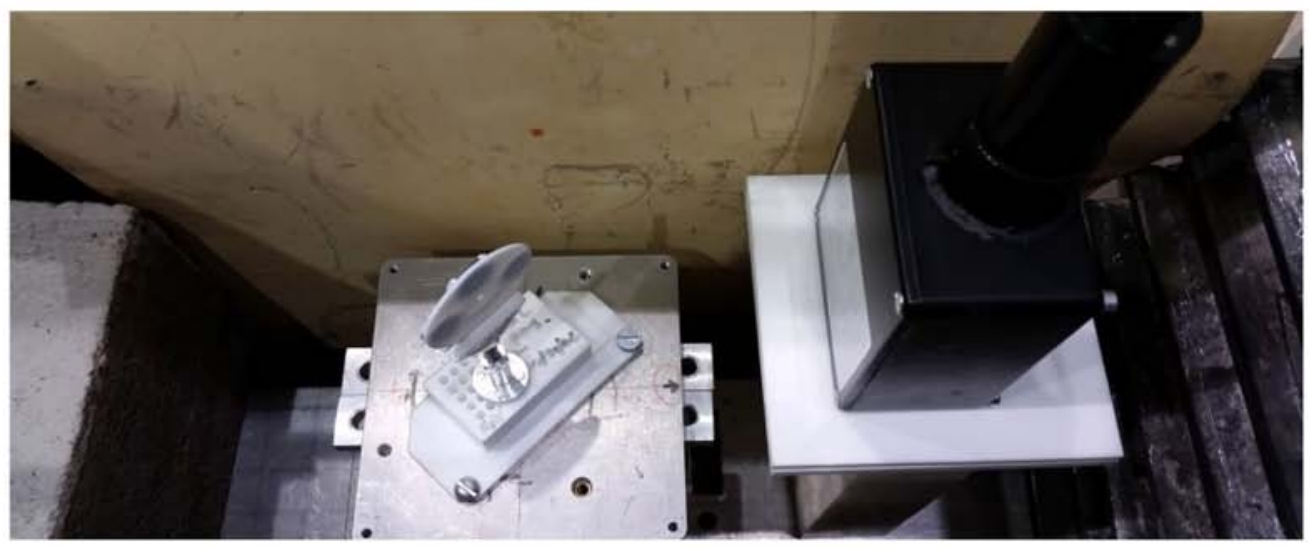

Fig. 1. Process of aligning each sample within neutron beam using 3D printed replicates of sample geometries with cadmium inserts and a neutron radiography camera

area of the boron carbide films ranged between 2.8 and $20.2 \mathrm{~cm}^{2}$ with a total sample mass between 0.4 and $1.82 \mathrm{~g}$ (including silicon substrate). Total sample thickness varied slightly in the sample set between 0.036 and $0.038 \mathrm{~cm}$, which also includes the silicon substrate. Using the net 
Table 1. Determined boron mass in each sample via aluminum as an external comparator element and silicon as an internal comparator element in PGAA measurements.

\begin{tabular}{lccc} 
& \multicolumn{2}{c}{ Boron mass $(\mu \mathrm{g})$ determined by } & \\
\cline { 2 - 3 } Sample number & $\begin{array}{c}\text { External } \\
\text { comparator }\left({ }^{7} \mathrm{Al}\right)\end{array}$ & $\begin{array}{c}\text { Internal } \\
\text { comparator }\left({ }^{28} \text { Si }\right)\end{array}$ & \% Difference \\
\hline 1 & $2886 \pm 133$ & $2847 \pm 134$ & 1.4 \\
2 & $2270 \pm 105$ & $2270 \pm 107$ & 0 \\
3 & $302 \pm 14$ & $298 \pm 14$ & 1.3 \\
4 & $1192 \pm 55$ & $1245 \pm 59$ & 4.4 \\
5 & $56 \pm 3$ & $56 \pm 3$ & 0 \\
6 & $36 \pm 2$ & $38 \pm 2$ & 5.6 \\
7 & $28 \pm 2$ & $29 \pm 1$ & 3.6 \\
8 & $36 \pm 2$ & $38 \pm 2$ & 5.6 \\
9 & $48 \pm 2$ & $48 \pm 2$ & 0 \\
10 & $3.1 \pm 0.3$ & $3.2 \pm 0.3$ & 3.2 \\
\hline
\end{tabular}

counts of the ${ }^{27} \mathrm{Al}(1779 \mathrm{keV})$ characteristic gamma ray from the comparator sample spectrum and net counts of the ${ }^{10} \mathrm{~B}(477 \mathrm{keV})$ and ${ }^{28} \mathrm{Si}$ (3539 keV) characteristic gamma rays from the boron carbide sample spectrum, the total boron mass was determined via two different reference elements for each sample. The results using aluminum as an external comparator and silicon as an internal comparator are presented and compared in Table 1.

A comparison of the total boron mass determined from the external aluminum comparator sample versus the internal silicon substrate content for each thin film analyzed in Table 1 clearly demonstrates a strong agreement between both approaches over a notable range of boron mass deposition. This supports initial assumptions that neutron and gamma-ray self-shielding effects within the sample are negligible in PGAA measurements of boron carbide thin films of similar characteristics to within the desired uncertainty. As further validation of this hybrid comparator method, the detector responses for the external aluminum comparator samples were also utilized in the same approach to calculate the mass of silicon substrate in each boron carbide sample. These results are compared with the known amount of silicon in each substrate in Fig. 2, which was disclosed shortly after the original PGAA experiments by the research team responsible for creating the boron carbide thin-film samples in the Department of Electrical and Computer Engineering at the University of Nebraska-Lincoln. 


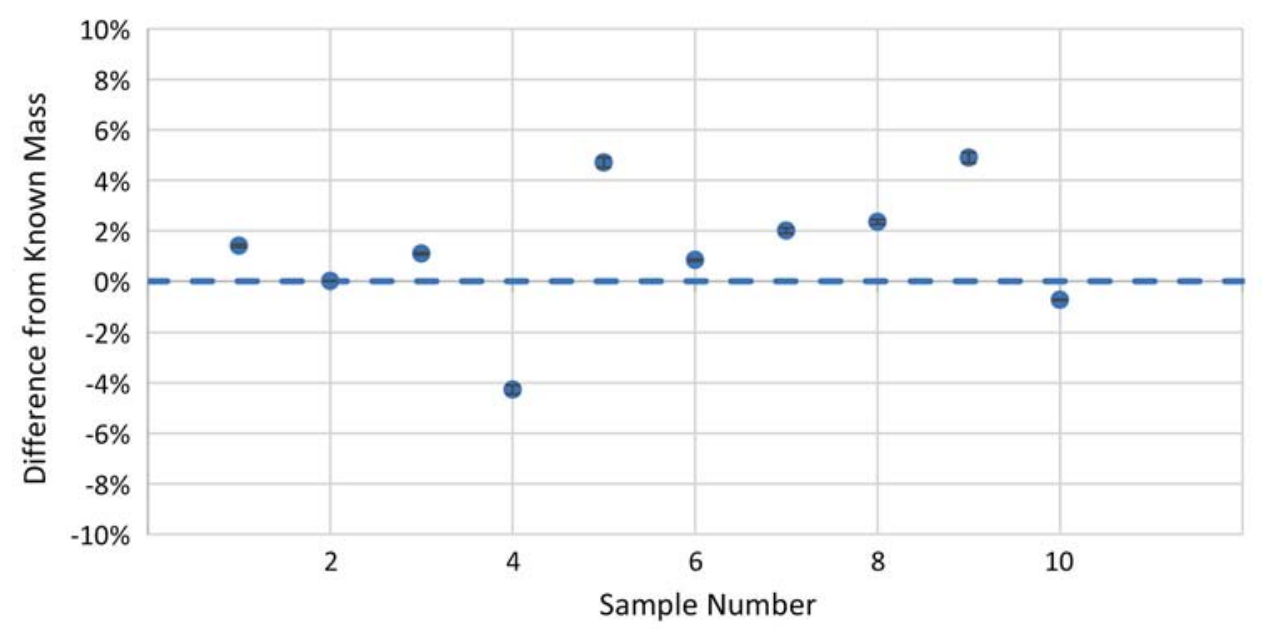

Fig. 2. Comparison of known silicon content in each silicon substrate with PGAA measurements using aluminum as the reference comparator material

Again, the results in Fig. 2 provide further confirmation of the boron mass results obtained using either the external aluminum comparator samples or internal silicon substrate content to standardize the PGAA boron measurements. As a final, more-direct effort to validate boron concentrations obtained using this method, an in-house standard with precisely measured boron content was created and analyzed using the same PGAA procedure with an aluminum external comparator sample having the same dimensions. The calculated boron mass (25.98 \pm 1.30$)$ was within $2.1 \%$ of the known boron mass (25.45 \pm 0.19 ), which demonstrates a sufficient level of accuracy in the measured boron content using this efficiency calibration method for the PGAA system.

\section{Conclusions}

A hybrid comparator method for boron calibration in PGAA measurements of boron carbide thin films deposited on silicon substrates has been introduced and benchmarked using two different implementations of a reference comparator material. The results show that accurate measurements of boron content in thin films are achievable to within approximately $5 \%$ accuracy of total boron mass. Also shown is the justification of initial assumptions made in simplifying the PGAA measurements using this method of analysis for boron carbide thin films of equivalent 
characteristics including negligible neutron and gamma-ray self-shielding effects, homogenous material matrix, and removal of spatially nonuniform flux dependence on calculations through the use of precise and repeatable sample placement within the incident neutron beam. This calibration method offers the capability of achieving accurate PGAA measurement of boron in thin films without the need to create an expensive reference standard for each sample of different geometry and may significantly reduce the turnaround time for completing the analysis of a large sample set. Significant promise in the application of this method for evaluating other thin-sample material compositions has also been demonstrated.

Although the accuracy of PGAA measurements in this study has only been confirmed to within approximately $5 \%$ of the calculated boron mass, it is believed that this method is capable of even higher levels of precision for boron as well as other elements. Future efforts are intended to reduce the uncertainties in the aforementioned measurements by reducing contributions from human error in sample placement and repeatability, inhomogeneity of boron in the thin film growths, uncertainty in concentration of comparator element in samples, and further addressing nonlinearities in the ratio of detector response to element mass due to the smaller influences in neutron and gamma ray self-shielding effects in thin-film samples. Other efforts will be directed upon the possibility of expanding this hybrid comparator approach to other thin-film elemental compositions.

Acknowledgments - We would like to thank the Office of Naval Research for providing the funding that made this project possible (Project Number N00014-16-1-2316 - DOD Navy Award\# 012800).

We also thank the staff at the Nuclear Engineering Teaching Laboratory at The University of Texas at Austin for their efforts in support of this research as well as the research team from the Department of Electrical and Computer Engineering at the University of Lincoln-Nebraska, who are responsible for the development and fabrication of the boron carbide thin film samples analyzed in this study.

\section{References}

1. Lindstrom RM, Révay Z (2017) Prompt gamma neutron activation analysis (PGAA): recent developments and applications. J Radioanal Nucl Chem 314:843858. https://doi.org/10.1007/s10967-017-5483-8 
2. Molnar G (2004) Handbook of Prompt Gamma Activation Analysis: With Neutron Beams. Springer, Berlin

3. Révay Z, Lindstrom RM, Mackey EA, Belgya T (2011) Neutron-induced prompt gamma activation analysis (PGAA). In: Vértes A, Nagy S, Klencsár Z et al (eds) Handbook of nuclear chemistry. Springer, Boston, pp 1619-1672

4. Paul RL (2005) Determination of boron in materials by cold neutron prompt gamma-ray activation analysis. Analyst 130:99. https://doi.org/10.1039/ $\underline{\mathrm{b} 412223 \mathrm{~b}}$

5. Biegalski SR, Green TC, Sayre GA et al (2005) Flux weighted efficiency calibration of the University of Texas at Austin PGAA Facility. J Radioanal Nucl Chem 265:303-308. https://doi.org/10.1007/s10967-005-0825-3

6. De Corte F, Simonits A, De Wispelaere A, Hoste J (1987) Accuracy and applicability of the k0-standardization method. J Radioanal Nucl Chem Artic 113:145-161. https://doi.org/10.1007/BF02036056

7. De Corte F, Bellemans F, Neve P, Simonits A (1994) The use of a modified Westcottformalism in the $\mathrm{k} 0$-standardization of NAA: the state of affairs. J Radioanal Nucl Chem 179:93-103. https://doi.org/10.1007/BF02037929

8. De Corte F (2000) k0and comparator NAA: influences and interactions. J Radioanal Nucl Chem 245:157-161. https://doi.org/10.1023/A:1006785417003

9. Paul RL (1995) The use of element ratios to eliminate analytical bias in cold neutron prompt gamma-ray activation analysis. J Radioanal Nucl Chem Artic 191:245-256. https://doi.org/10.1007/BF02038220

10. Molnár GL, Révay Z, Paul RL, Lindstrom RM (1998) Prompt-gamma activation analysis using the k0 approach. J Radioanal Nucl Chem 234:21-26. https://doi. org/10.1007/BF02389741

11. Révay Z, Harrison RK, Alvarez E et al (2007) Construction and characterization of the redesigned PGAA facility at The University of Texas at Austin. Nucl Instrum Methods Phys Res Sect A Accel Spectrom Detect Assoc Equip 577:611-618. https://doi.org/10.1016/j.nima.2007.04.119

12. Ríos-Martínez C, Ünlü K, Wehring BW (1998) Performance of the University of Texas cold-neutron prompt gamma activation analysis facility. J Radioanal Nucl Chem 234:119-123. https://doi.org/10.1007/BF02389758

13. Alvarez E, Biegalski SR, Landsberger S (2007) Methodologies for hydrogen determination in metal oxides by prompt gamma activation analysis. Nucl Instrum Methods Phys Res Sect B Beam Interact Mater Atoms 262:333-339. https://doi.org/10.1016/j.nimb.2007.06.004

14. Magara M, Yonezawa C (1998) Decomposition of prompt gamma-ray spectra including the Doppler-broadened peak for boron determination. Nucl Instrum Methods Phys Res Sect A Accel Spectrom Detect Assoc Equip 411:130-136. https://doi.org/10.1016/S0168-9002(98)00303-9

15. Szentmiklósi L, Gméling K, Révay Z (2007) Fitting the boron peak and resolving interferences in the 450-490 keV region of PGAA spectra. J Radioanal Nucl Chem 271:447-453. https://doi.org/10.1007/s10967-007-0229-7 
16. Harrison RK, Landsberger S (2009) Determination of boron over a large dynamic range by prompt-gamma activation analysis. Nucl Instrum Methods Phys Res Sect B Beam Interact Mater Atoms 267:513-518. https://doi.org/10.1016/j. nimb.2008.11.057

17. Biegalski SR, Green TC, Alvarez E, Aghara S (2007) Background characterization of The University of Texas PGAA Facility. J Radioanal Nucl Chem 271:413-417. https://doi.org/10.1007/s10967-007-0224-Z

18. Höglund C, Zeitelhack K, Kudejova P et al (2015) Stability of 10B4C thin films under neutron radiation. Radiat Phys Chem 113:14-19. https://doi. org/10.1016/j.radphyschem.2015.04.006 